\title{
Use of Numerical Model and Nonlinear Regression in Determining Thermo-Material Properties of Thermal Barrier Coatings Using Flash Thermography
}

\author{
by L. Sripragash*, M. Goldammer** and M. Koerdel* \\ * Siemens Inc., Charlotte, NC, USA, letchuman.sripragash@siemens.com, martin.koerdel@siemens.com \\ ** Siemens AG Corporate Technology, Munich, Germany, matthias.goldammer@siemens.com
}

\begin{abstract}
Determination of thermo-material properties of Thermal Barrier Coatings (TBC) is critical in gas turbine industry. In this study, with the aid of a numerical model and non-linear regression, material properties of the TBC were determined [1], [2]. The effect of thicknesses of second and third layers on the thermographic responses were also analysed. A precalculated grid concept was used for the estimation of material properties. A significant increase in computational speed was observed when pre-calculated grid data was used for regression analysis.
\end{abstract}

\section{Introduction}

Flash thermographic non-destructive testing (FTNDT) is one of the rapid non-contact non-destructive testing method used for inspection of critical components and rapid inspection of large areas. General classification of active thermographic technique is based on the kind of heat source used, the method how heat is applied on the surface (eg. pulse, wave form or step heat etc.) and the image processing technique used [3]. In flash thermography a heat pulse is applied on the surface of the object to be inspected using an optical flash lamp, and the surface temperature is monitored using an infrared (IR) camera. Analysis of surface temperature with time is helpful in identifying embedded irregularities of the object being inspected. Temperature evolution of the surface of an object due to heat energy applied at the surface can be better understood through heat conduction principles. Though, IR camera captures the IR intensities, in thermographic techniques, for the range of temperatures at the surface of the object, temperature is assumed to be linearly related to the IR intensity. In flash thermography there are two major kinds of data processing techniques available, namely image-based and pixel-based processing techniques. In image-based technique contrast images are used for defect characterization, and in pixel-based technique the thermographic profile of selected pixels is analysed. In quantitative analysis of flash thermography, as proposed by Shepard et al in their Thermographic Signal Reconstruction (TSR) technique [4] time derivatives of the thermal profile in logarithmic domain of pixels also found to be useful.

Thermographic response for multilayer material system is useful in characterizing thermal barrier coatings used in gas turbine industries. Balageas et al [1] provided theoretical background and analytical solutions for multi-layered materials with various flash conditions. Once the experimental data obtained theoretical solutions can be used with a regression analysis to identify layered material properties. Numerical models will be useful when theoretical solutions are unavailable or becomes complicated. Sun and Tao [5] have used regression analysis with numerical model to estimate properties of multi-layered material system. In their regression analysis, they minimized the error in the temperature profile between the actual and numerical profiles to estimate the best numerical model parameters. They also introduced a weighing function to increase the influence of inflection points in the regression analysis.

This study focusses on the use of FTNDT in characterizing multi-layered materials, especially the Thermal Barrier Coatings (TBC) used in gas turbine industries, with the use of a numerical model and regression analysis. In this study for regression analysis, second derivative $(2 \mathrm{~d})$ evolution was used in a selected region where inflection point occurs on the temperature profile. The choice of $2 \mathrm{~d}$ evolution simplifies the analysis, as it does not need an accurate value for the heat flux applied at the surface to generate numerical data. Also, the use of $2 \mathrm{~d}$ evolution enabled automatic regression as the $2 d$ curve can be used to identify the inflection point from extrema points. In addition, numerical data were created a priori for fast computation purpose called "pre-calculated grids".

\section{Background}

\subsection{Theory}

As described in the previous section, FTNDT is mainly depending on the heat conduction of the object being inspected. General heat conduction equation can be given by

$$
\nabla \cdot(K \cdot \nabla T)+\dot{q}=\rho c \frac{\partial T}{\partial t}
$$

where $K, T, \dot{q}, \rho, c$ and $t$ are thermal conductivity matrix, absolute temperature, internal heat generation rate per volume, density, specific heat capacity and time respectively. In usual FTNDT, the heat diffusion is assumed to be one 
dimensional (1D) as the test specimen are of plate like structures. Though the assumption may not be valid close to the defects and near any irregularities such as inclusion of different material, voids etc., the deviation of the heat diffusion from the ideal 1D case would usually give information on the characteristics of defects. The governing equation for flash thermographic analysis can be reduced to the following one-dimensional heat equation,

$$
\alpha \frac{d^{2} T}{d z^{2}}+\frac{\dot{q}}{\rho c}=\frac{d T}{d t}
$$

where $\alpha$ is the diffusivity of the material and is given by

$$
\alpha=\frac{k}{\rho c}
$$

There are analytical solutions available to the above equations for various material systems and flash conditions [1] [6]. In this study an in house developed numerical model is used for the analysis [7] and the procedure have been improved by incorporating regression analysis for better quantitative analysis.

In quantitative analysis of FTNDT, it is found that derivative images and pixel wise evolution profiles are much useful [4]. In this study derivatives are used to identify the interface between the layers through the inflection points. Accordingly, first and second derivatives ( $1 \mathrm{~d}$ and $2 \mathrm{~d}$ ) are defined by

$$
1 d=\frac{d[\log (\Delta T)]}{d[\log (t)]}
$$

and

$$
2 d=\frac{d^{2}[\log (\Delta T)]}{d[\log (t)]^{2}}
$$

In multilayered materials system, from two inflection points on the temparature evolution curve at least two of the parameters can be extracted [8] and they are thermal thickness $\left(\mu_{t h}\right)$ given by

$$
\mu_{t h}=\frac{l}{\sqrt{\alpha}}
$$

and the reflectivity $(\mathrm{R})$ or the thermal mismatch factor at the interface between layer 1 and 2 is given by [9]

$$
R=\frac{\varepsilon_{2}-\varepsilon_{1}}{\varepsilon_{2}+\varepsilon_{1}}
$$

Where $\varepsilon_{i}$ is the effusivity or thermal intertia of the $i^{\text {th }}$ layer material defined as

$$
\varepsilon_{i}=\sqrt{k_{i} c_{i} \rho_{i}}
$$

\subsection{Numerical modelling}

The model used in this study was developed using Finite Difference solution methods. The heat equations are solved using Crank-Nicholson Algorithm (see for example [10]). The developed model has been validated for two-layers system, and finite flash duration effects using analytical results [7]. In this study the model has been used in combination with regression analysis to provide property maps. In summary the governing equation given in equation (2) can be discretised using Crank Nicholson method and is given by,

$$
\alpha\left\{\frac{\left(T_{i+1}^{j+1}-2 T_{i}^{j+1}+T_{i-1}^{j+1}\right)+\left(T_{i+1}^{j}-2 T_{i}^{j}+T_{i-1}^{j}\right)}{2 \Delta z^{2}}\right\}+\frac{Q_{i j}}{\rho c}=\frac{\left(T_{i}^{j+1}-T_{i}^{j}\right)}{\Delta t}
$$

Where $i$ and $j$ represent the spatial node location and the time step respectively. This equation can be solved following Crank-Nicholson algorithm via finite difference solution technique. Once the heat equation is solved for temperatures on each time step, $1 d$ and $2 d$ data can also can be created. In this study since the focus is on extracting material properties of TBC, the interface resistance between layers are neglected.

\subsection{Regression analysis}

It is observed that in layered material the point of inflection and the curvature at the inflection point are related to the thermal thickness and reflectivity. Therefore, in this study, we strict ourselves to extract only two of the top layer properties based on the first inflection point after the flash effect. The best fit method utilizes a set of results of known materials and geometries to identify the two unknown properties of the top layer of the actual object. The best fit was found by minimizing the norm value between the actual data and a set of data from a data base of known properties. 


\section{Methodology}

The overall procedure implemented in extracting the necessary properties can be described using the flowchart given in Fig. 1. The experimental setup used in this study is shown in Fig. 2. It contains a mid-wave IR camera (FLIR SC5000), Hensel flash lamp (Helsel EH PRO 6000), flash generation unit (Helsel TRIA 6000 S) and a computer for data acquisition. The experimental specimen used was a sample created with three different thickness of thermal barrier coatings $(0.56 \mathrm{~mm}, 1.2 \mathrm{~mm}$, and $2.2 \mathrm{~mm})$. The data acquisition was done with the internally developed Siemens proprietary software $\operatorname{SInE}^{2}$ (Siemens Infrared series Explorer) and the rest of the analysis is carried out using MATLAB.

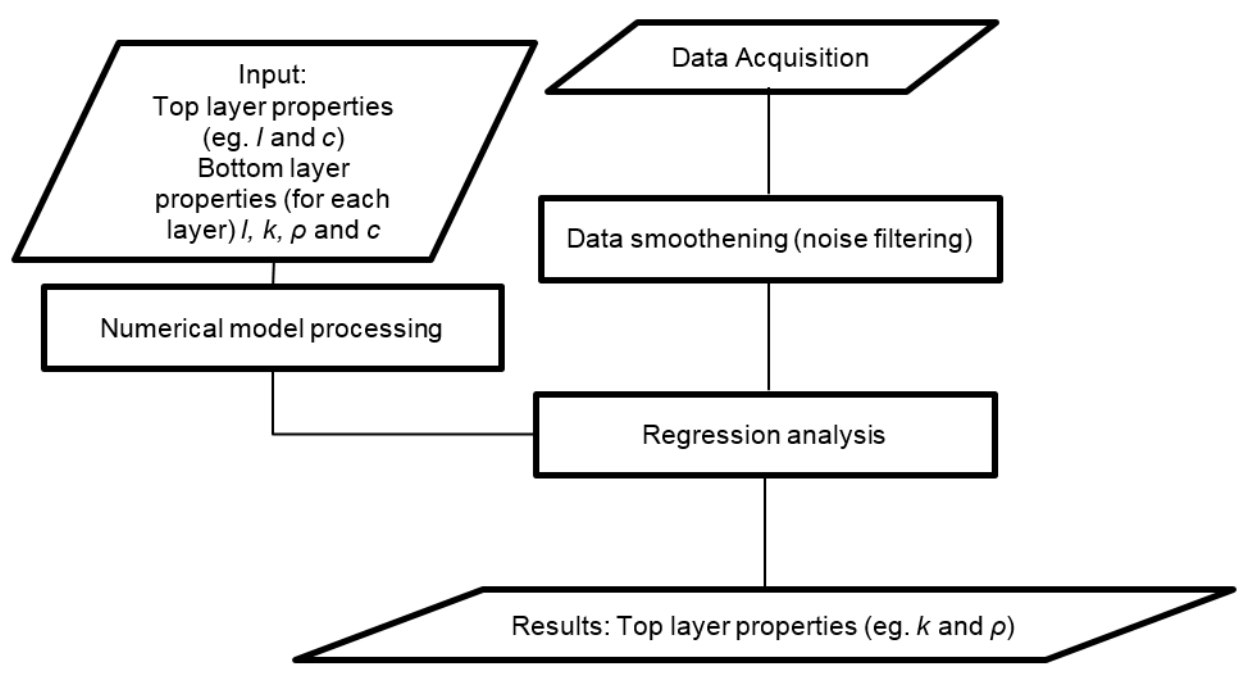

Fig. 1. Methodology implemented

The data acquired from IR camera is usually pre-processed to extract meaningful information as it contains noise. Pre-processing includes pre-flash correction, data smoothening and obtaining derivatives. Regression analysis can be performed for temperature data, $1 \mathrm{~d}, 2 \mathrm{~d}$ data or any other form of data based on temperature evolution. A set of preprocessed thermographic profiles are given in Fig. 3. In this study, from an initial trial and error analysis, compared to the temperature data and $1 \mathrm{~d}$ data, $2 \mathrm{~d}$ data found to be more appropriate for regression analysis.

The numerical model solutions can be obtained for any number of layers and different kinds of applied flash. In this study it was observed that the flash duration effects can be neglected as the inflection points observed were obtained at relatively later time for the thicknesses of the top layers used. Therefore, Dirac pulse have been implemented in numerical model. The input parameters necessary are the bottom layer material and geometric properties namely thickness, conductivity, specific heat capacity and density. In addition, some of the numerical parameters also been selected such as the number of elements in each layer, time steps, boundary condition, duration and if a different flash type is chosen then the necessary flash parameters.

In the regression analysis, the inflection points on the thermographic evolution curves have been utilized to extract necessary properties. In that case it would be enough to perform regression analysis in a locally selected region of the evolution curve [2]. In this study, since $2 d$ thermographic profile have been identified as a parameter to be used for regression analysis, the region where the negative peak occurs have been identified for regression analysis. Identifying the negative peak of the $2 d$ would be a straight forward aspect. In the logarithmic time domain, it is possible to define a window size in such a way that can be universally implemented. The window size is an approximate time interval in logarithmic domain, in such a way that the midpoint of the window would be the point where negative peak of $2 \mathrm{~d}$ occurs. The total size of the window is selected in such a way that the influence of the inflection point is captured. Fig. 4 shows the resultant full range window selected in the logarithmic time domain. This aspect enabled us for automating the regression analysis.

Once the relevant data for regression was selected, in this case $2 d$ data with specified window, non-linear regression analysis can be performed directly to extract unknowns. But it is comparatively time consuming if we need to extract the unknown properties for all the pixels from the thermal image. Therefore, it is decided to use non-linear regression analysis only to estimate the range of unknown properties. The two properties to be estimated are the two axes of the grids to be created and for each grid point numerical thermographic profiles are obtained. This created pre-calculated grid forms a data base of known properties of thermographic profiles. The thermographic profile obtain from real components can be then compared with the data base to extract unknown properties using regression analysis. Fig. 4 shows a sample of the best fit of numerical results and the experimental results for the selected window of the $2 \mathrm{~d}$ data. 


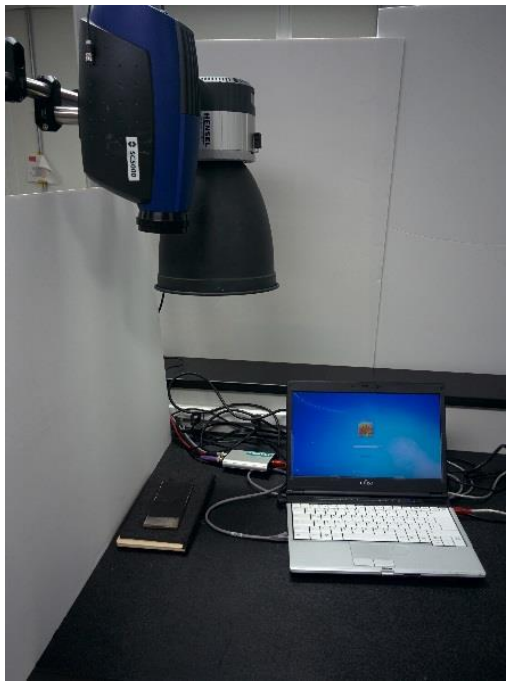

Fig. 2: Experimental Setup

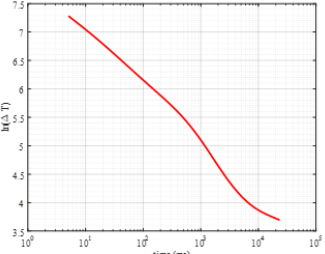

(a)

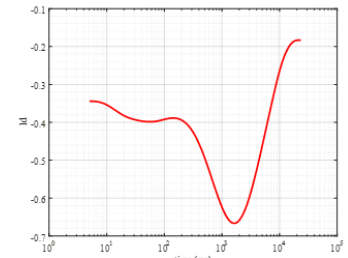

(b)

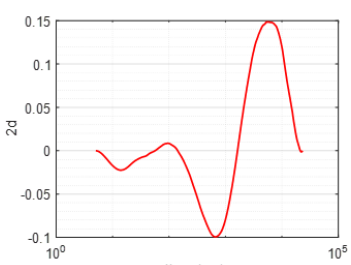

(c)

Fig. 3. Sample of pre-processed thermographic profiles (a) Temperature (b) 1d and (c) $2 d$ evolutions

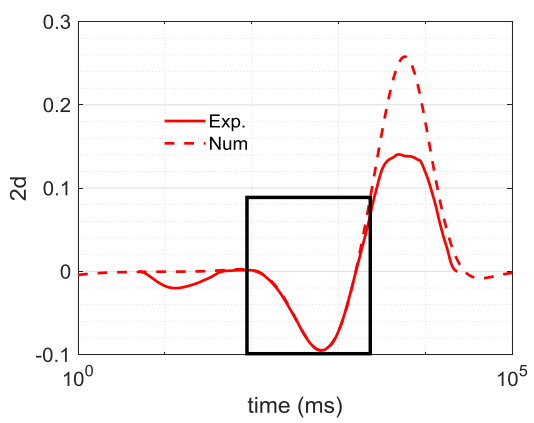

Fig. 4: Best-fit numerical and experimental $2 d$ curves

\section{Analysis}

\subsection{Thickness and conductivity map}

The following analysis is done to extract thickness and conductivity values of the specimen used. Referring to Fig. 1 if thickness and conductivity are to obtain as output on the top most layer, then specific heat capacity and density are supposed to be input parameters. It is found that the density was varying based on the plasma spray parameters used in the TBC process. One of the thermal images for the specimen used in this study and the corresponding locations for which $2 d$ thermographic profiles are obtained for the nonlinear regression are shown in Fig. 5 (a). The geometrical and material properties assumed in this analysis are given in Table 1. Thermographic profiles extracted for these three locations and the corresponding $2 \mathrm{~d}$ profiles obtained using nonlinear regression and numerical model are given in Fig. 5(b). 
Table 1. Thickness and material properties used for the analysis given in Fig. 5

\begin{tabular}{|l|l|l|l|}
\hline & TBC (top layer) & Bond coating (middle layer) & Substrate (Bottom layer) \\
\hline$l / \mathrm{mm}$ & -- & 0.2 & 6 \\
\hline$k /(\mathrm{W} / \mathrm{m} / \mathrm{K})$ & -- & 5.0 & 10.0 \\
\hline$\rho /\left(\mathrm{kg} / \mathrm{m}^{3}\right)$ & 6050 & 7500 & 7500 \\
\hline$c /(\mathrm{J} / \mathrm{kg} / \mathrm{K})$ & 537 & 480 & 493 \\
\hline
\end{tabular}

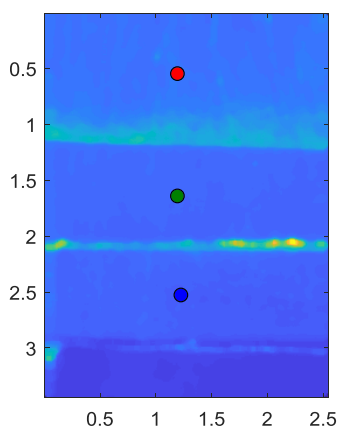

(a)

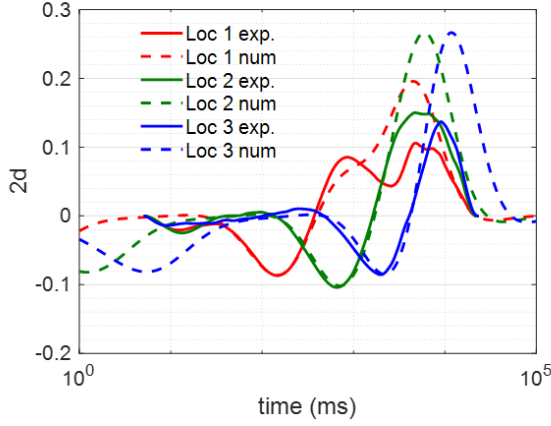

(b)

Fig. 5: Non-linear regression results (a) locations selected for the analysis (b) comparison of corresponding $2 d$ evolution curves

Since the nonlinear regression consumes time to extract the unknown parameters, pre-calculated grids have been generated for the length and conductivity as being grid points as shown in Fig. 6 . In generating such grid points the first step is to estimate the properties, in this case thickness and the conductivity, from a single measured curve using nonlinear regression as shown by a dark black dot in Fig. 6 . Then having the estimation, a possible range of properties are assumed, and thermographic profiles are generated with sufficient interval in the property values to fill the grid. Each grid point in the grid shown in Fig. 6 (a) consists a second derivative thermographic profile as shown in Fig 6(b).

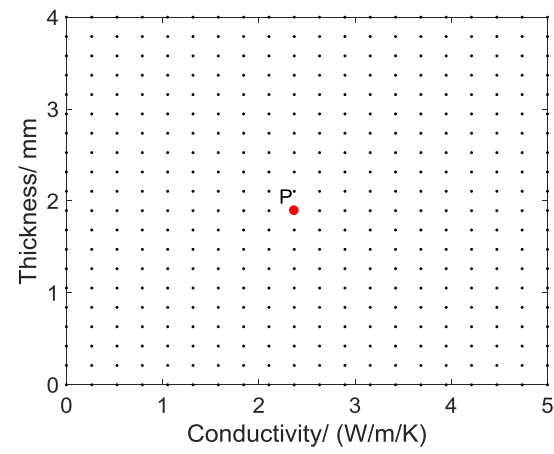

(a)

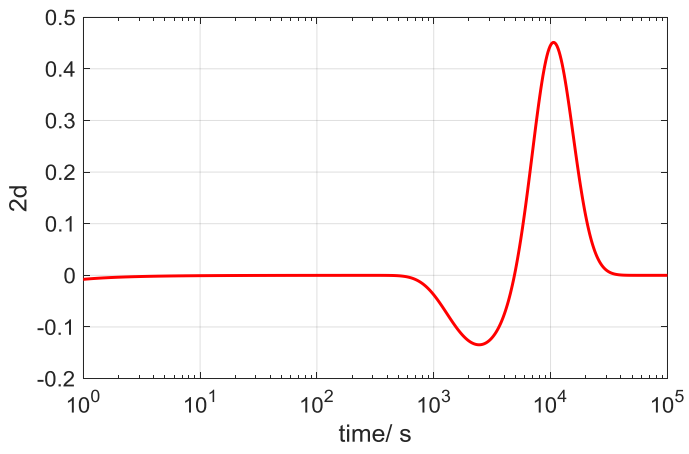

(b)

Fig. 6: (a)Pre-calculated grid (b) a sample profile representing the grid point $P$ given in (a)

The experimental thermographic profiles obtained for each pixel are then compared with the created thermographic profiles of the pre-calculated grids by calculating the mean squared difference. In this case the minimum of these norm values have been used to estimate the best matching position in the pre-calculated grid to give the properties for the experimental curve. In this study it was found that the creation of pre-calculated grid result in a significant computational speed. In a same computer with the pre-calculated grid, regression found to be about 100 times faster than the direct nonlinear regression.

\subsection{Influence of substrate and bond-coat thickness}

FTNDT is influenced by the equipment used, the image acquisition settings, geometrical parameters and the thermomaterial properties of the object being inspected. In this section a numerical analysis is done to describe the influence of the bond-coat (second layer) and substrate (third layer) thicknesses in a TBC system. The influence of the bond coating and substrate thicknesses in the thermographic evolution are given in Fig. 7 and Fig. 8 respectively. The thickness and material properties used for these analyses are given in 
Table 2 and Table 3 . The influence of the bond coating is found to be minimal compared to the influence of the substrate thickness for the range of values selected.

Table 2: Thickness and material properties used for the analysis of bond coat thickness variation

\begin{tabular}{|l|l|l|l|}
\hline & TBC & Bond coating & Substrate \\
\hline$l / \mathrm{mm}$ & 1 & $0.1,0.2,0.5$ & 5 \\
\hline$k /(\mathrm{W} / \mathrm{m} / \mathrm{K})$ & 1 & 10 & 9 \\
\hline$\rho /\left(\mathrm{kg} / \mathrm{m}^{3}\right)$ & 5000 & 7500 & 8500 \\
\hline$c /(\mathrm{J} / \mathrm{kg} / \mathrm{K})$ & 500 & 500 & 400 \\
\hline
\end{tabular}

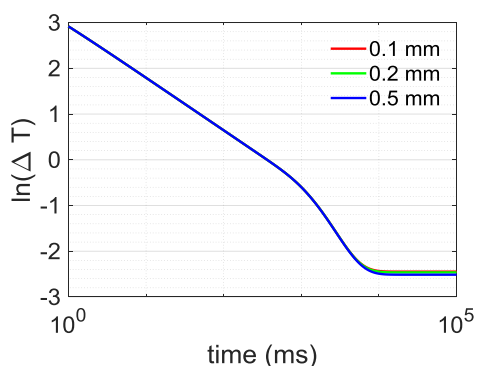

(a)

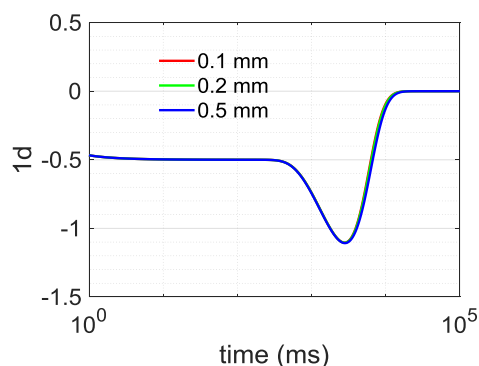

(b)

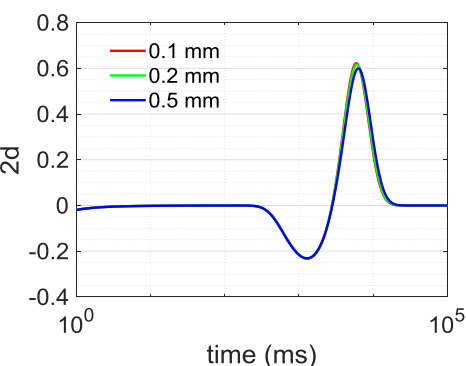

(c)

Fig. 7. The influence of bond-coat thickness on (a) temperature (b) $1 d$ and (c) $2 d$ thermographic profiles

Table 3. Thickness and material properties used for the analysis of substrate thickness variation

\begin{tabular}{|l|l|l|l|}
\hline & TBC & Bond coating & Substrate \\
\hline$l / \mathrm{mm}$ & 1 & 0.2 & $1,2,4,5,10$ \\
\hline$k /(\mathrm{W} / \mathrm{m} / \mathrm{K})$ & 1 & 10 & 9 \\
\hline$\rho /\left(\mathrm{kg} / \mathrm{m}^{3}\right)$ & 5000 & 7500 & 8500 \\
\hline$c /(\mathrm{J} / \mathrm{kg} / \mathrm{K})$ & 500 & 500 & 400 \\
\hline
\end{tabular}
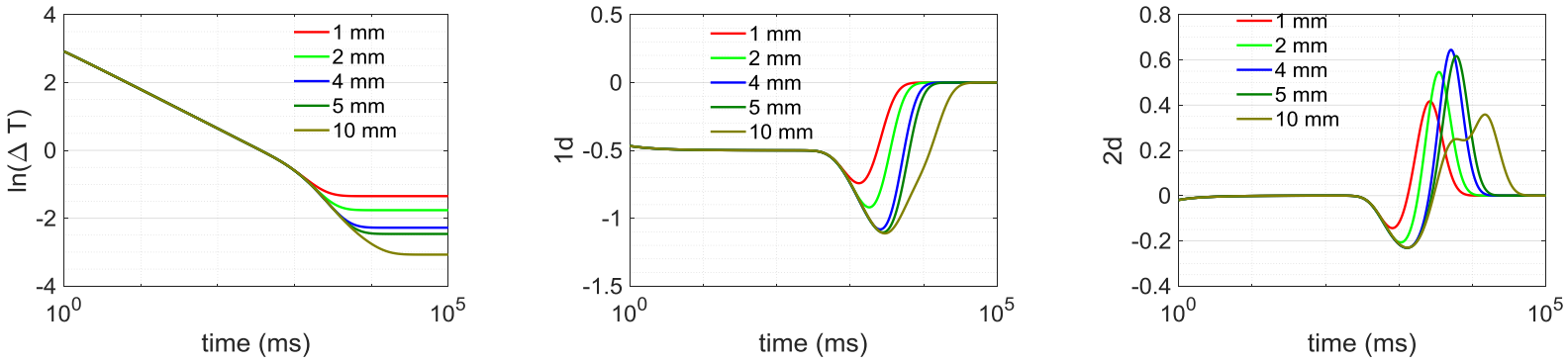

Fig. 8. The influence of substrate thickness on (a) temperature (b) $1 d$ and (c) $2 d$ thermographic profiles

\section{Results}

Thickness and conductivity map are displayed in Fig. 9 and they are found using pre-calculated grid data. As stated earlier, to obtain the thickness and conductivity map, the specific heat capacity and density values of the top layer considered to be constants. In TBC process, density is a parameter expected to be estimated, it will be useful if the density map can be provided as an output. Therefore currently, the possibility of including thickness data as an input is being investigated. Thickness data can be obtained from 3D scanning methods. The object on which the thickness is needed first scanned before and after coating. The difference between the scan data would provide a thickness map and this map can be used to provide conductivity and density map as output parameters. A set of results obtained from nonlinear regression analysis on estimating conductivity and density is provided in Fig. 10 and the numerical values of the results are given in Table 4. 

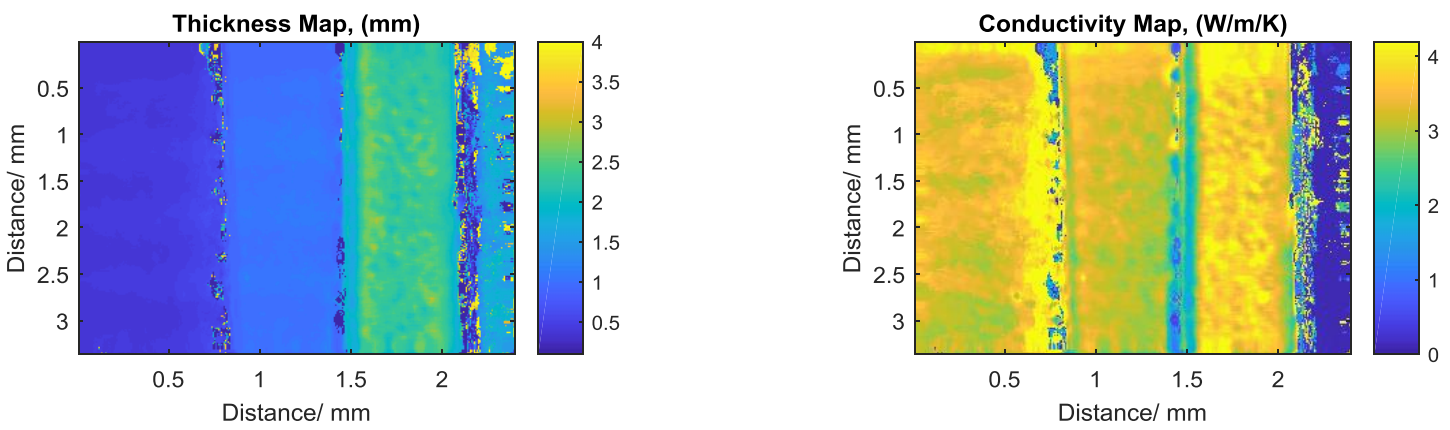

Fig. 9. Property maps (a) thickness and (b) conductivity
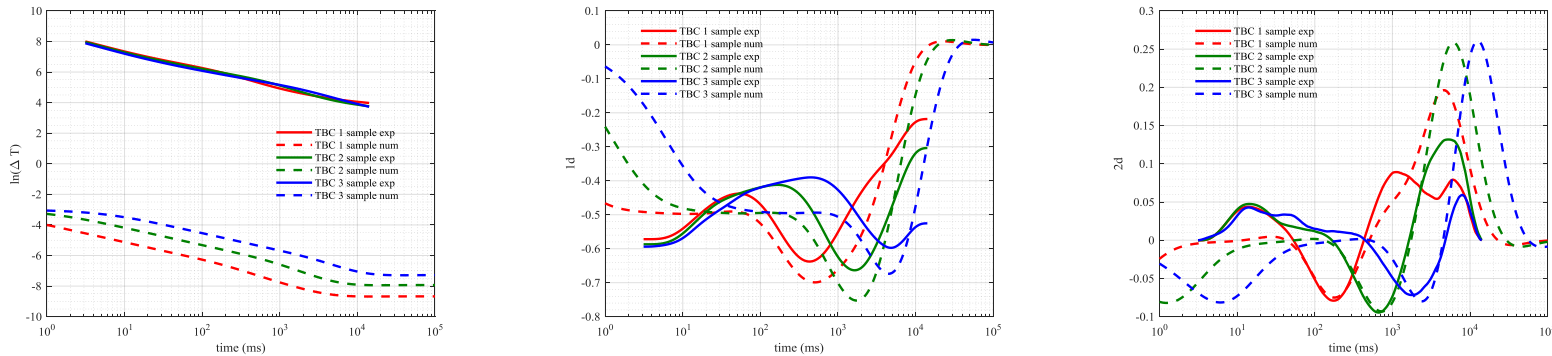

Fig. 10. Regression results obtained for unknown density and conductivity

Table 4. Properties estimated from regression analysis

\begin{tabular}{|r|r|}
\hline Conductivity $/(\mathrm{W} / \mathrm{m} / \mathrm{K})$ & Density $/\left(\mathrm{kg} / \mathrm{m}^{3}\right)$ \\
\hline 5.00 & 5300 \\
\hline 3.62 & 5644 \\
\hline 3.10 & 7000 \\
\hline
\end{tabular}

\section{Conclusion}

Two of the unknown parameters on the top layer of layered material have been estimated using flash thermography. The use of $2 \mathrm{~d}$ data in regression analysis enabled successful extraction of two unknown properties. The local region selected for the regression was successfully automated, with the use of a window size in logarithmic time interval, and the negative peak point as an inflection point. In addition, the success of the procedure depending on the following factors

1. Accuracy of the material properties provided for the rest of the layers.

2. The thermal properties of the layers should be significantly different, and the layers should be thermally thick enough in such a way to clearly see inflection point.

3. The TBC found to be IR transparent and therefore graphite coating is necessary if a mid-wave IR camera is used. Therefore, to make this procedure successful, a longwave camera to be used.

Extraction of density and conductivity as output results is possible if the thickness of the top layer is provided. Therefore, the study will be continued to use 3D scan data for thickness measurement to obtain density and conductivity maps from flash thermographic response.

\section{REFERENCES}

[1] D. L. Balageas, J. C. Krapez, and P. Cielo, "Pulsed photothermal modeling of layered materials," J. Appl. Phys., vol. 59, no. 2, pp. 348-357, 1986.

[2] J. G. Sun, "Pulsed Thermal Imaging Measurement of Thermal Properties for Thermal Barrier Coatings Based on a Multilayer Heat Transfer Model," J. Heat Transfer, vol. 136, no. 8, p. 81601, 2014.

[3] C. Ibarra-Castanedo, J. R. Tarpani, and X. P. V Maldague, "Nondestructive testing with thermography," Eur. J. Phys., vol. 34, pp. S91-S109, 2013.

[4] S. M. Shepard, J. R. Lhota, B. A. Rubadeux, D. Wang, and T. Ahmed, "Reconstruction and enhancement of active thermographic image sequences," Opt. Eng., vol. 42, no. 5, pp. 1337-1342, 2003. 
[5] J. G. Sun and N. Tao, "Thermal property measurement for thermal barrier coatings using pulsed thermal imaging - multilayer analysis method," vol. 100004, p. 100004, 2016.

[6] H. S. Carslow and J. C. Jaeger, Conduction of Heat in Solids, Second Edi. Ne: Clarendon Press Oxford, 1986.

[7] L. Sripragash, M. Goldammer, and M. Kördel, "Numerical Modeling and Comparison of Flash Thermographic Response," Thermogr. 2017, 2017.

[8] J. Baumann, M. Goldammer, and W. Heinrich, "Method for determining Material parameters of an object from temperature-versus-time (t-t) data," 2006.

[9] M. Xavier P. V., Theory and Practice of Infrared Technology for Nondestructive Testing. New York: John Wiley \& Sons, Inc., 2001.

[10] D. Greenspan, Discrete Numerical Methods in Physics and Engineering. New York: Academic Press Inc., 1974. 